\title{
Principais conclusões do workshop conjunto dos programas FAPESP BIOTA-BIOEN-Mudanças Climáticas: ciência e políticas públicas para uma economia mais verde, no contexto da $\mathrm{RIO}+20$
}

\author{
Carlos Alfredo Joly ${ }^{1,8}$, Roberto Gomes de Souza Berlinck ${ }^{2}$, Vanderlan da Silva Bolzani, \\ Célio Fernando Baptista Haddad ${ }^{4}$, Mariana Cabral de Oliveira ${ }^{5}$, Marie-Anne Van Sluys, \\ Glaucia Mendes Souza ${ }^{6}$, Luciano Martins Verdade ${ }^{7}$ \& Reynaldo Luiz Victoria ${ }^{7}$ \\ ${ }^{1}$ Departamento Biologia Vegetal, Instituto de Biologia - IB, Universidade Estadual de \\ Campinas - UNICAMP, Barão Geraldo, CP 6109, CEP 13081-970, Campinas, SP, Brasil \\ ${ }^{2}$ Instituto de Química de São Carlos, Universidade de São Paulo - USP, CP 780, \\ CEP 13560-970, São Carlos, SP, Brasil \\ ${ }^{3}$ Instituto de Química de Araraquara, Universidade Estadual Paulista - UNESP, CP 355, \\ CEP 14800-900, Araraquara, SP, Brasil \\ ${ }^{4}$ Instituto de Biociências, Universidade Estadual Paulista - UNESP, Av. 24A, 1515, Bela Vista, \\ CP 199, CEP 13506-900, Rio Claro, SP, Brasil \\ ${ }^{5}$ Instituto de Biociências, Universidade de São Paulo - USP, R. do Matão, Travessa 14, 321, \\ CEP 05508-900, São Paulo, SP, Brasil \\ ${ }^{6}$ Instituto de Química, Universidade de São Paulo - USP, Av. Prof. Lineu Prestes, 748, \\ CEP 05508-900, São Paulo, SP, Brasil \\ ${ }^{7}$ Centro de Energia Nuclear na Agricultura, Universidade de São Paulo - USP, Av. Pádua Dias, 11, \\ CEP 13416-000, Piracicaba, SP, Brasil \\ ${ }^{8}$ Autor para correspondência: Carlos Alfredo Joly, e-mail: cjoly@unicamp.br
}

JOLY, C.A., BERLINCK, R.G.S., BOLZANI, V.S., HADDAD, C.F.B., OLIVEIRA, M.C., VAN SLUYS, M-A., SOUZA, G.M., VERDADE, L.M. \& VICTORIA, R.L. Main conclusions of the joint FAPESP Programs BIOTA-BIOEN-Climate Change Workshop: Science and Policy for a Greener Economy in the context of RIO+20. Biota Neotrop. 12(2): http://www.biotaneotropica.org.br/v12n2/en/item?point-of-view+bn00112022012

\begin{abstract}
This Point of View summarizes the conclusion of a joint workshop organized by FAPESP's Environmental Research Programs - BIOTA (The Virtual Institute of Biodiversity) - BIOEN (Bioenergy Research) - Climate Change, to discuss the scientific contribution to the RIO+20 UN Conference. Brazilian scientists gathered at FAPESP's headquarters in São Paulo City in the first week of March 2012 raised the following concerns: a) that there are very few opportunities for the scientific community to interact with Conferences like the RIO+20; b) the deficiencies of the ZeroDraft, document produced by UN's Division for Sustainable Development for the RIO+20; c) the fact that the focus of all three research programs - biodiversity, bioenergy and climate change - are not on the agenda of discussions of RIO +20; d) that little emphasis is given to the oceans in the Conference Agenda; e) regarding market mechanisms associated with the transition to a greener economy, the need to emphasize the reduction of perverse subsidies and promotion of economic incentives to activities or processes to mitigate emissions and/or carbon sequestration; f) that in Brazil we need to stimulate and consolidate research focused on Ecosystem Services Assessments and Evaluation. The workshop participants recognized the need to strengthen knowledge about international conventions, treaties and agreements signed and ratified by Brazil, as well as international institutions, programs and initiatives to promote the participation of the scientific community in global environmental policies debate. Finally, from the viewpoint of the three FAPESP programs two points were highlighted: a) that it is imperative to deepen the scientific knowledge in each of the three focal areas - biodiversity, bioenergy and climate change - because it is necessary to increase the critical mass of researchers and knowledge to reach the global forum in these strategic areas; b) it is also imperative to support and promote research projects that integrate the focal areas of the three programs, bringing together inter and transdisciplinary teams. This is a worldwide trend in the area of global environmental change, and participants of the three programs feel they can make a significant contribution to the advancement of knowledge, to the international debate and for the effective solution of problems.
\end{abstract}

Keywords: RIO+20, sustainability, green economy, environmental research, global change.

JOLY, C.A., BERLINCK, R.G.S., BOLZANI, V.S., HADDAD, C.F.B., OLIVEIRA, M.C., VAN SLUYS, M-A., SOUZA, G.M., VERDADE, L.M. \& VICTORIA, R.L. Principais Conclusões do Workshop conjunto dos Programas FAPESP BIOTA-BIOEN-Mudanças Climáticas: ciência e políticas públicas para uma economia mais verde, no contexto da RIO+20. Biota Neotrop. 12(2): http://www.biotaneotropica.org.br/v12n2/ pt/item?point-of-view+bn00112022012

Resumo: Este Ponto de Vista resume as conclusões de um Workshop conjunto, organizado pelos três Programas da FAPESP na Área Ambiental - BIOTA (O Instituto Virtual da Biodiversidade) - BIOEN (Pesquisa em 
Bioenergia) - Mudanças Climáticas, para discutir a contribuição da comunidade científica para a RIO+20, a Conferência das Nações Unidas para o Desenvolvimento Sustentável. O grupo de pesquisadores brasileiros reunidos pela FAPESP no início de março de 2012 levantou as seguintes preocupações: a) o número reduzido de oportunidades para a comunidade científica interagir com Conferências como a RIO+20; b) as graves deficiências do ZeroDraft, documento produzido pela Divisão das Nações Unidas para o Desenvolvimento Sustentável para a RIO +20; c) o fato do foco de pesquisa dos três Programas de Pesquisa Ambiental da FAPESP - biodiversidade, bioenergia e mudanças climáticas - não estarem na pauta das discussões da RIO+20; d) que pouca ênfase é dada aos oceanos na Agenda da Conferência; e) em relação aos mecanismos de mercado associados com a transição para uma economia mais verde, a necessidade de enfatizar a redução de subsídios perversos e a promoção de incentivos econômicos para atividades ou processos de mitigação e/ou sequiestro de carbono; f) a necessidade de estimular o desenvolvimento e a consolidação da pesquisa na área de avaliação e valoração de serviços ambientais, no Brasil. Os participantes do Workshop reconheceram a necessidade de aprofundar o conhecimento sobre as convenções, tratados e acordos internacionais assinados e ratificados pelo Brasil, bem como as instituições internacionais, programas e iniciativas que promovem a participação da comunidade científica no debate de políticas ambientais globais. Finalmente, do ponto de vista dos três programas da FAPESP dois pontos foram destacados: a) que é imperativo aprofundar o conhecimento científico em cada uma das três áreas focais - biodiversidade, bioenergia e mudanças climáticas - porque é necessário aumentar a massa crítica de pesquisadores e do conhecimento para participar das discussões internacionais nessas áreas estratégicas; b) também é imperativo apoiar e promover projetos de pesquisa que integrem as áreas focais dos três programas, estimulando a constituição de equipes inter e transdisciplinares. Esta é uma tendência mundial na área das mudanças ambientais globais, e os participantes dos três programas sentem que podem dar uma contribuição significativa para o avanço do conhecimento, para o debate internacional e para a efetiva solução dos problemas.

Palavras-Chave: $R I O+20$, sustentabilidade, economia verde, pesquisa ambiental, mudanças ambientais globais.

\section{Introdução}

Em 2012, o Brasil hospeda a Conferência das Nações Unidas em Desenvolvimento Sustentável (UNCSD), ou RIO+20, a ser realizada de 20 a 22 de junho no Rio de Janeiro. A Secretaria-Executiva da Comissão Nacional para a Conferência das Nações Unidas sobre Desenvolvimento Sustentável é constituída pelos Ministérios das Relações Exteriores, da Fazenda, do Desenvolvimento Social e Combate à Fome, e Ministério do Meio Ambiente. A Comissão Nacional da RIO+20 propriamente dita, tem uma composição mais abrangente, envolvendo 20 ministérios, entre os quais o de Ciência, Tecnologia e Inovação, além de instituições relevantes da comunidade científica, como a ABC, SBPC e representantes da sociedade civil e empresarial (vide Decreto No 7.495, de 7 de junho de 2011).

A resolução das Nações Unidas estabelece que o objetivo da conferência é a "renovação do compromisso político com o desenvolvimento sustentável, por meio da avaliação do progresso na implementação dos resultados das Cúpulas anteriores sobre Desenvolvimento Sustentável (Rio, 1992; Johanesburgo, 2002) e do tratamento de temas novos e emergentes". Além disso, a Rio+20 tratará dos seguintes dois temas: "economia verde no contexto do desenvolvimento sustentável e da erradicação da pobreza" e "estrutura institucional do desenvolvimento sustentável".

Como parte do processo preparatório, o Brasil, assim como todos os demais países membros das Nações Unidas, encaminhou à ONU em 01/11/2011 sua contribuição para o documento síntese a ser discutido na conferência de junho. Em janeiro de 2012 a ONU publicou a primeira versão do documento síntese (denominado ZeroDraft), reunindo as contribuições de todos os países e organizações por ela reconhecidas. Desde então este documento tem balizado a preparação do documento final da RIO+20, que será subscrito pelos chefes de estado presentes no Rio.

A FAPESP, visando contribuir para o processo da RIO+20, e ampliar a possibilidade de pesquisadores brasileiros expressarem suas posições em relação a estes temas, realizou nos dia 07 e 08 de março de 2012 o BIOTA-BIOEN-Climate Change Joint Workshop: Science and Policy for a Greener Economy in the context of RIO+20.

Este Ponto de Vista resume as principais conclusões dos participantes deste workshop.

\section{Resultados}

1) Os participantes mostraram preocupação com as poucas oportunidades para discussões relativas à temática de desenvolvimento científico, tecnológico e inovador como estratégia para uma transição para uma economia mais verde, no âmbito da RIO+20.

2) Os participantes foram unânimes em apontar deficiências no documento ZeroDraft, produzido pela Divisão para o Desenvolvimento Sustentável da ONU. Os principais problemas apontados foram:

a) não reconhecer que há um limite natural na capacidade suporte do planeta, e que ações para equilibrar a demanda por alimentos, recursos naturais (incluindo água) e energia, até a metade do século XXI, precisam ser tomadas agora;

b) não reconhecer que o equilíbrio do pilar ambiental é condição sine qua non para que os outros dois pilares da sustentabilidade, o social e o econômico, possam se desenvolver;

c) a sustentabilidade ambiental é condição sine qua non para garantir acesso a condições dignas de vida e desenvolvimento e para erradicação da pobreza, pois a instabilidade ambiental afeta de forma distinta o planeta, prejudicando mais as populações mais pobres;

d) praticamente não há menção a um projeto de educação, peçachave no processo de transição para uma economia mais verde, que implica em mudanças de hábitos de consumo e estilo de vida; e

e) pouca ênfase é dada ao conhecimento produzido pelo desenvolvimento científico, tecnológico e inovador, estratégico na reformulação da economia atual para uma economia mais verde. 
3) Os participantes mostraram preocupação com o fato do foco de pesquisa dos três Programas de Pesquisa Ambiental da FAPESP - biodiversidade, bioenergia e mudanças climáticas - não estarem na pauta das discussões da RIO+20;

4) Identificou-se também, especificamente, o pequeno destaque dado aos oceanos, fonte de recursos essenciais para a sobrevivência de milhões de pessoas, fronteira ainda pouco conhecida em relação à biodiversidade, e reconhecida como de fundamental importância na questão das mudanças ambientais globais;

5) Na avaliação dos mecanismos de mercado associados à transição para uma economia mais verde, foi enfatizada a necessidade de redução de subsídios perversos, por exemplo, a redução da alíquota de impostos sobre automóveis sem uma exigência de contrapartida de eficiência e/ou redução na emissão de gases, bem como da possibilidade de taxação de carbono, por exemplo, a taxa de permissão de emissão de $\mathrm{CO}_{2}$ recentemente implantada pela União Européia às companhias aéreas. Mas a ênfase maior foi dada ao favorecimento, em alguns casos através de incentivos fiscais, às atividades de mitigação de emissões e/ou sequestro de carbono.

6) Ênfase foi dada também à questão da valoração dos serviços ambientais, reconhecendo-se que esta é uma área na qual o país ainda carece de conhecimento científico. Trata-se, portanto, de uma área de pesquisa que precisa de apoio para expansão e consolidação no Brasil.

7) Avaliação dos resultados do questionário on-line “ $A$ participação dos pesquisadores dos programas BIOEN, BIOTA e Mudanças Climáticas na RIO+20", evidencia a necessidade dos pesquisadores dos três programas conhecerem mais profundamente convenções, tratados e acordos assinados e ratificados pelo Brasil, bem como instituições, programas e iniciativas internacionais, que promovem a participação da comunidade científica no debate internacional.

8) Finalmente, e do ponto de vista dos três Programas FAPESP, talvez o mais relevante seja destacar dois aspectos quanto à necessidade estratégica das pesquisas na área de mudanças ambientais globais:

a) de um lado é imperativo aprofundarmos o conhecimento científico em cada uma das três áreas focais - biodiversidade, bioenergia e mudanças climáticas - porque é necessário aumentar a massa crítica de pesquisadores e de conhecimento, para uma atuação mais estratégica nestas áreas;

b) por outro, considerando-se as mudanças ambientais globais, é igualmente imperativo o apoio e o incentivo a projetos de pesquisa que integrem as áreas focais dos três Programas, reunindo equipes inter e transdisciplinares. A reunião de pesquisadores, de diversas formações e áreas de atuação, em torno do equacionamento de questões ambientais concretas, é fundamental dada à multiplicidade de atores e de impactos. A necessidade do desenvolvimento de projetos com estas características segue uma tendência mundial na área de mudanças ambientais globais, e os três Programas avaliam que podem dar uma contribuição significativa para o avanço do conhecimento, para o debate internacional e para a efetiva solução de problemas.

\section{Leituras Complementares Recomendadas}

HALONEN, T., ZUMA, J., AL NAHAYAN, S.A.Z., AZ-ZUBAIR, H.A., BABACAN, A., BALSILLIE, J.L., BEDRITSKY, A., BRUNDTLAND, G.H., CALMY-REY, M., LILLO, J.C., CARLSSON, G., DIOGO, L.D., SEUNG-SOO, H., HATOYAMA, Y., HEDEGAARD, C., RUIZ, C.N., RAMESH, J., RICE, S.E., RUDD, K., STUART, F., TEIXEIRA, I.M.V. \& GUOGUANG, Z. 2012. Resilient People, Resilient Planet: A Future Worth Choosing. The Secretariat for the High-level Panel on Global Sustainability, New York, United Nations. www.un.org/gsp.

INTERGOVERNMENTAL OCEANOGRAPHIC COMMISSION OF UNESCO - IOC/UNESCO, INTERNATIONAL MARITIME ORGANISATION - IMO, FOOD AND AGRICULTURE ORGANIZATION OF UNITED NATIONS - FAO \& UNITED NATIONS DEVELOPMENT PROGRAMME - UNDP. 2011. A Blueprint for Ocean and Coastal Sustainability. IOC/UNESCO, Paris.

INTERNATIONAL COUNCIL FOR SCIENCE - ICSU, DIVERSITAS, INTERNATIONAL GEOSPHERE-BIOSPHERE PROGRAMME - IGBP, INTERNATIONAL HUMAN DIMENSIONS PROGRAMME ON GLOBAL ENVIRONMENTAL CHANGE - IHDP, WORLD CLIMATE RESEARCH PROGRAMME - WCRP \& EARTH SYSTEM SCIENCE PARTNERSHIP - ESSP. 2012. The State of the Planet Declaration. In: Planet Under Pressure: New Knowledge Towards Solutions Conference. ICSU, London. http://www. planetunderpressure2012.net/pdf/state_of_planet_declaration.pdf.

PLANET UNDER PRESSURE: New Knowledge Towards Solutions Conference. 2012. Policy Briefs. Elsevier. Titles: Water security; Food security; Biodiversity and ecosystems; Transforming governance and institutions; Interconnected risks and challenges; Energy security; Health; Well-being; Green economy. http://www.planetunderpressure2012.net/ policybriefs.asp.

PROGRAMA DAS NAÇÕES UNIDAS PARA O MEIO AMBIENTE - PNUMA, 2011. Caminhos para o Desenvolvimento Sustentável e a Erradicação da Pobreza - Síntese para Tomadores de Decisão. PNUMA. www.unep.org/greeneconomy. 